\title{
A Robust Nonlinear Output Feedback Control Method ' for Limit Cycle Oscillation Suppression Using Synthetic Jet Actuators
}

\author{
Natalie Ramos Pedroza ${ }^{1}$, William MacKunis ${ }^{1}$, and Vladimir Golubev ${ }^{2}$ \\ ${ }^{1}$ Physical Sciences Department, Embry-Riddle Aeronautical University, Daytona Beach, Florida 32114 \\ ${ }^{2}$ Aerospace Engineering Department, Embry-Riddle Aeronautical University, Daytona Beach, Florida 32114
}

\begin{abstract}
A synthetic jet actuator-based output feedback control method is presented, which achieves asymptotic limit cycle oscillation regulation in small unmanned aerial vehicle wings, where the dynamic model contains uncertainty and unmodeled external disturbances. In addition, the proposed control method compensates for the parametric uncertainty and nonlinearity inherent in the synthetic jet actuator dynamics. Motivated by the limitations characteristic of small unmanned aerial vehicles, the control method is designed to be computationally inexpensive, eliminating the need for time-varying parameter update laws, function approximators, or other computationally heavy techniques. To this end, a computationally minimal robust-inverse control method is utilized, which is proven to compensate for the uncertainties in both the aerial vehicle dynamics and the synthetic jet actuator dynamics. By endowing the robust-inverse control law with a bank of dynamic filters, asymptotic limit cycle oscillation regulation is achieved using only pitching and plunging displacement measurements in the feedback loop. The result is an asymptotic synthetic jet actuator-based limit cycle oscillation regulation control method, which does not require velocity measurements, adaptive laws, or function approximators in the feedback loop. To achieve the result, a detailed mathematical model of the limit cycle oscillation dynamics is utilized, which includes nonlinear stiffness effects, unmodeled external disturbances, and dynamic model uncertainty, in addition to the parametric uncertainty in the synthetic jet actuator dynamic model. A rigorous Lyapunov-based stability analysis is utilized to prove asymptotic regulation of limit cycle oscillations, and numerical simulation results are provided to demonstrate the performance of the proposed control law.
\end{abstract}

\section{INTRODUCTION}

The design of limit cycle oscillation suppression systems is extremely important in aircraft applications, since limit cycle oscillations can cause dynamic instability that could result in catastrophic damage [1], [2]. To ease readability in this paper, the following four acronyms will be defined: limit cycle oscillations (LCO); synthetic jet actuators (SJA); small unmanned aerial vehicle (SUAV); and angle of attack (AoA). LCO, or flutter, is characterized as pitching (rotational) and plunging (vertical) displacements in an airfoil. A synthetic jet actuator (SJA)-based LCO suppression method is presented in this paper, which is designed to be practically implementable in small unmanned aerial vehicle (SUAV) flight applications, where onboard space and computational power is limited.

LCO suppression systems are usually designed based on the assumption that the full state (i.e., pitching and plunging displacement and velocity measurements) is available for feedback [3], [4], [5], [6], [7], [8]. Although the availability of velocity measurements is a standard assumption, velocity information can be difficult to obtain accurately due to system faults and/or low sensor measurement resolution [9]. Motivated by this fact, eliminating the need for velocity measurements is important in LCO suppression system design [10]. Moreover, the inherent space limitations involved in SUAV applications motivates the development of control laws that can be implemented with a reduced computational cost and space requirement.

To reduce cost, weight, and mechanical complexity over standard mechanical control surfaces (e.g., ailerons and elevators), SJAs are becoming increasingly popular in SUAV control applications. SJAs transfer linear momentum to a flow system using a piezoelectric membrane inside a cavity, which creates trains of air vortices through the alternating ejection and suction of air through a small orifice. A key benefit of SJA is that they are capable of achieving momentum transfer with zero net mass flux across the flow boundary. For this reason, SJAs do not require space for a fuel supply, and this feature reduces the space and weight requirements when used in SUAV applications. These SJAs can be utilized to achieve LCO suppression by modifying the boundary layer flow field near the surface of an aircraft wing. SJAs can also improve SUAV maneuverability by expanding the usable range of angle of attack (AoA) [11]. However, one of the challenges in SJA-based control design is that the SJA actuator dynamic model is nonlinear and includes parametric uncertainty. For further details regarding SJA operation and modeling, readers are referred to [12], [13], [14], [15] and the references therein.

The contribution in this paper is a SJA-based output feedback control method, which achieves asymptotic LCO regulation in SUAV wings in the presence of uncertain SUAV dynamics and unmodeled external disturbances. In addition, the proposed control method compensates for the parametric uncertainty and nonlinearity inherent in the SJA actuator dynamics. Motivated by the limitations characteristic of SUAV applications, the control method is designed to be computationally inexpensive, eliminating the need for time-varying parameter update laws, function approximators, or heavy computations. To this end, a robust-inverse control method [12] is utilized, which is proven to compensate for the SUAV and SJA uncertainties using a simplified controller structure. By endowing the robust-inverse control structure with with a bank of dynamic filters, asymptotic 
LCO regulation is achieved using only pitching and plunging displacement measurements in the feedback loop. The result is an asymptotic SJA-based LCO regulation control design, which does not require velocity measurements, adaptive laws, function approximators, or heavy computations in the feedback loop. To achieve the result, a detailed mathematical model of the LCO dynamics is utilized, which includes nonlinear stiffness effects, unmodeled external disturbances, and SUAV model uncertainty. An additional challenge addressed in the control design is the parametric uncertainty and nonlinearity that is inherent in the SJA dynamic model. A rigorous Lyapunov-based stability analysis is utilized to prove the theoretical result, and numerical simulation results are provided to demonstrate the performance of the proposed control law.

\section{Dynamic Model And PRoperties}

In this section, a detailed mathematical model of the pitching and plunging dynamics in an airfoil will be presented, which incorporates nonlinear stiffness effects, unmodeled nonlinear external disturbances, and the uncertain nonlinear SJA actuator dynamics. To facilitate the control design, the LCO dynamics will be expressed in an advantageous form, which will be utilized to design the LCO suppression control law.

The equation describing LCO in an UAV wing can be expressed as [16]

$$
M_{s} \ddot{p}+C_{s} \dot{p}+F(p) p+d(t)=\left[\begin{array}{l}
-F_{L} \\
M
\end{array}\right]
$$

where the coefficients $M_{s}, C_{s} \in \mathbb{R}^{2 \times 2}$ are the structural mass and damping matrices; $F(p(t)) \in \mathbb{R}^{2 \times 2}$ is a nonlinear stiffness matrix; and $p(t) \triangleq\left[\begin{array}{ll}h(t) & \alpha(t)\end{array}\right]^{T} \in \mathbb{R}^{2}$ denotes the state vector, where $h(t), \alpha(t) \in \mathbb{R}$ denote the plunging $[m]$ and pitching $[\mathrm{rad}]$ displacements, respectively. In (1), $d(t) \in \mathbb{R}^{2}$ represents a general unknown, norm-bounded, nonvanishing disturbance.

Assumption 1. The disturbance $d(t)$ is bounded and sufficiently smooth such that $d(t), \dot{d}(t) \in \mathcal{L}_{\infty}$ throughout closed-loop operation.

Property 1. The structural mass matrix $M_{s}$ is positive definite and symmetric (see [16] and [17]).

In (1), the structural linear mass, $M_{s}$, structural linear damping, $C_{s}$, and the nonlinear stiffness, $F(p)$, matrices are described as [16]

$$
M_{s}=\left[\begin{array}{cc}
m & m x_{\alpha} b \\
m x_{\alpha} b & I_{\alpha}
\end{array}\right], C_{s}=\left[\begin{array}{cc}
C_{h} & 0 \\
0 & C_{\alpha}
\end{array}\right], F(p)=\left[\begin{array}{cc}
K_{h} & 0 \\
0 & K_{\alpha}(\alpha)
\end{array}\right]
$$

where $x_{\alpha} \in \mathbb{R}$ denotes the non-dimensional distance measured from the elastic axis to the center of mass, $b \in \mathbb{R}$ is the semichord of the wing $[\mathrm{m}], m \in \mathbb{R}$ is the mass of the wing section $[\mathrm{kg}]$, and $I_{\alpha} \in \mathbb{R}$ is the mass moment of inertia of the wing about the elastic axis $\left[\mathrm{kg} \cdot \mathrm{m}^{2}\right]$. The parameter $C_{h} \in \mathbb{R}$ denotes the structural damping coefficient in plunge due to viscous damping $[\mathrm{kg} / \mathrm{s}]$, and $C_{\alpha} \in \mathbb{R}$ denotes the structural damping coefficient in pitch due to viscous damping $\left[\mathrm{kg} \cdot \mathrm{m}^{2} / \mathrm{s}\right]$. The $K_{h} \in \mathbb{R}$ is the structural spring constant in plunge $[\mathrm{N} / \mathrm{m}]$; and $K_{\alpha}(\alpha(t)) \in \mathbb{R}$ is the nonlinear torsion stiffness coefficient $[\mathrm{N} \cdot \mathrm{m} / \mathrm{rad}]$, which is defined via the polynomial

$$
K_{\alpha}=2.82\left(1-22.1 \alpha+1315.5 \alpha^{2}-8580 \alpha^{3}+17289.7 \alpha^{4}\right) .
$$

Remark 1. The exact polynomial definition for the nonlinear torsion stiffness coefficient in (3) is provided for completeness in defining the dynamic model only [17]. The polynomial in (3) is assumed to be unknown and is not used in the control design. The proposed robust nonlinear control law compensates for the uncertainty associated with nonlinear torsion stiffness.

Also in (1), the control force $F_{L}(t) \in \mathbb{R}$ and control moment $M(t) \in \mathbb{R}$ are defined as

$$
\begin{aligned}
F_{L} & =\rho U^{2} s_{p} b c_{l_{\alpha}}\left[\alpha+\frac{\dot{h}}{b}+\left(\frac{1}{2}-a\right) b \frac{\dot{\alpha}}{U}\right]+\rho U^{2} s_{p} b c_{l_{\beta}} \beta \\
M & =\rho U^{2} s_{p} b^{2} c_{m_{\alpha}}\left[\alpha+\frac{\dot{h}}{b}+\left(\frac{1}{2}-a\right) b \frac{\dot{\alpha}}{U}\right]+\rho U^{2} s_{p} b^{2} c_{m_{\beta}} \beta
\end{aligned}
$$

where $U \in \mathbb{R}$ denotes forward velocity $[\mathrm{m} / \mathrm{s}], s_{p} \in \mathbb{R}$ is the wing span $[\mathrm{m}], c_{l_{\alpha}} \in \mathbb{R}$ is the lift coefficient per angle of attack, $c_{m_{\alpha}} \in \mathbb{R}$ is the moment coefficient per control surface deflection, $c_{l_{\beta}} \in \mathbb{R}$ is the lift coefficient per control surface deflection, $c_{m_{\beta}} \in \mathbb{R}$ is the moment coefficient per control surface deflection, and $a \in \mathbb{R}$ is the non-dimensional distance from the mid-chord to the elastic axis. In (4) and (5), the term $\beta(t) \in \mathbb{R}$ denotes the control surface deflection [deg].

Property 2. The control surface deflection $\beta(t)$ in (4) and (5) will be generated by means of SJA arrays. In the following Section II-A, the nonlinear dynamic model for the virtual surface deflection due to arrays of SJAs will be described. To simplify the following discussion, it will be assumed that the virtual surface deflection is generated by $m=2$ arrays of SJAs; however, the control design can be easily extended to handle any number $m \geq 2$ SJA arrays with little modification (e.g., using the pseudo-inverse of a matrix).

After some rearranging of (1), the LCO dynamics can be expressed as

$$
M_{s} \ddot{p}=\chi(t)-d(t)+B u
$$


where the unknown, unmeasurable, nonlinear auxiliary signal $\chi(t) \in \mathbb{R}^{2}$ is defined as

$$
\chi(t) \triangleq-C_{s} \dot{p}-F(p) p
$$

where $F(p)$ is the nonlinear stiffness. In (6), $u(t) \triangleq\left[\begin{array}{ll}u_{1}(t) & u_{2}(t)\end{array}\right]^{T} \in \mathbb{R}^{2}$ denotes the virtual surface deflection angle due to the SJA arrays; and $B \in \mathbb{R}^{2 \times 2}$ is an uncertain control input gain matrix. To facilitate the following discussion, the constant elements of the control input gain matrix will be denoted as

$$
B=\left[\begin{array}{ll}
B_{1,1} & B_{1,2} \\
B_{2,1} & B_{2,2}
\end{array}\right]
$$

In the definition given in (8), the $i^{t h}$ column of $B$ for $i=1,2$ contains the aerodynamic parameters $B_{1, i}, B_{2, i}$ corresponding to the $i^{\text {th }}$ SJA array. The parameters $B_{1, i}$ and $B_{2, i}$ for $i=1,2$ are explicitly defined as [16]

$$
B_{1, i}=\left(\rho v^{2} b^{2} c_{m \beta} s_{p}+\frac{I_{\alpha}}{m x_{a} b} \rho v^{2} b c_{l \beta} s_{p}\right)_{i}, \quad B_{2, i}=\left(-\rho v^{2} b c_{l \beta} s_{p}-\frac{1}{x_{a} b} \rho v^{2} b^{2} c_{m \beta} s_{p}\right)_{i} .
$$

\section{A. Virtual Surface Deflection Due to SJAs}

For scenarios when the control actuation is generated by a given number, $i$, of SJA arrays, the control input term $u(t)$ in Equation (6) physically represents the virtual deflection angle resulting from the combined control effort of the SJAs. In this case, the virtual deflection angle resulting from the $i^{\text {th }}$ SJA array is a nonlinear function of the voltage applied to that array. Based on experimental data, the virtual surface deflection due to the $i^{\text {th }}$ SJA array can be expressed using the empirically determined model [13], [14], [15]

$$
u_{i}(t)=\theta_{2, i}^{*}-\frac{\theta_{1, i}^{*}}{v_{i}(t)}, \quad i=1,2
$$

where $u_{i}(t) \in \mathbb{R}$ denotes the virtual airfoil surface deflection due to the $i^{t h}$ SJA array; $v_{i}(t) \triangleq A_{p p i}^{2}(t) \in \mathbb{R}$, where $A_{p p i}$ denotes the peak-to-peak voltage amplitude that is applied to the $i^{\text {th }}$ SJA array in [Volts]; and $\theta_{1, i}^{*}, \theta_{2, i}^{*} \in \mathbb{R}$ are uncertain constant physical parameters in [Volt-deg] and [deg], respectively (for further details on the SJA model in (10), readers are referred to [12], [13], [14], [15]). Note that, physically, the parameter $\theta_{2, i}^{*}$ represents the maximum surface deflection angle achievable using the $i^{\text {th }}$ SJA array.

To compensate for the SJA actuator nonlinearity and input parametric uncertainty in (10), a robust-inverse control structure is utilized [12], which employs constant, "best-guess" estimates of the uncertain SJA parameters $\theta_{1}^{*}, \theta_{2}^{*}$. The robust-inverse control law can be expressed as

$$
v_{i}(t)=\frac{\hat{\theta}_{1, i}}{\hat{\theta}_{2, i}-u_{d i}(t)}, \quad i=1,2
$$

where $\hat{\theta}_{1, i}, \hat{\theta}_{2, i} \in \mathbb{R}$ are the constant feedfoward estimates of $\theta_{1, i}^{*}, \theta_{2, i}^{*}$; and $u_{d i}(t) \in \mathbb{R}$ is a subsequently defined auxiliary control signal.

Remark 2 (Avoiding Singularities). Based on (11), the control signal $v_{i}(t)$ will encounter singularities when $u_{d i}(t)=\hat{\theta}_{2, i}$. To avoid these singularities in the control law, the control signals $u_{d i}(t)$ will be designed using the algorithm [18]

$$
u_{d i}(t)=\left\{\begin{array}{cl}
\hat{\theta}_{2, i}-\epsilon & \text { if } g\left(\mu_{0}, \mu_{1}\right) \geq \hat{\theta}_{2, i}-\epsilon \\
g\left(\mu_{0}, \mu_{1}\right) & \text { otherwise }
\end{array}\right.
$$

where $\epsilon \in \mathbb{R}^{+}$is a small parameter; $g(\cdot)$ is a subsequently defined control function; and $\mu_{0}(t), \mu_{1}(t) \in \mathbb{R}^{2}$ are subsequently defined auxiliary feedback control terms. Note that the parameter $\epsilon$ can be selected arbitrarily small, so that system stability can be proven over an arbitrarily large range of positive control voltage signals $v_{i}(t)$.

In addition, the control terms $u_{i}(t)$ in (10) will encounter singularities when $v_{i}(t)=0$, which occurs when $\hat{\theta}_{1, i}=0$ for any $i$. However, the singularity at $v_{i}(t)=0$ can be easily avoided by selecting $\hat{\theta}_{1, i}$ based on the knowledge that $\hat{\theta}_{1, i}>0$ for $i=1,2$ [13], [14], [15].

After substituting (10) and (11) into (6), the open-loop dynamics are obtained as

$$
M_{s} \ddot{p}=\chi(t)-d(t)+\Omega u_{d}
$$

where $u_{d}(t) \triangleq\left[u_{d 1}(t), u_{d 2}(t)\right]^{T} \in \mathbb{R}^{2}$, and $\Omega \in \mathbb{R}^{2 \times 2}$ denotes a constant uncertain matrix. Specifically, $\Omega$ contains the uncertain terms from the input gain matrix $B$ in addition to the uncertain SJA parameters $\theta_{1, i}^{*}$ for $i=1,2$.

III. CONTROL DEVELOPMENT

The objective is to design the control signal $u_{d}(t)$ to regulate the plunging and pitching dynamics (i.e., $h(t), \alpha(t)$ ) to zero. To quantify the control objective, a LCO regulation error $e(t) \in \mathbb{R}^{2}$ and auxiliary error signal $r(t) \in \mathbb{R}^{2}$ are defined as

$$
\begin{gathered}
e=p-p_{d} \\
r=\dot{e}+\phi e+e_{f}
\end{gathered}
$$


where $\phi \in \mathbb{R}^{2 \times 2}$ is a positive definite, diagonal control gain matrix; the desired plunging and pitching states $p_{d} \triangleq\left[h_{d}, \alpha_{d}\right]^{T}=$ $[0,0]^{T}$ for the LCO suppression objective; and $e_{f}(t) \in \mathbb{R}^{2}$ denotes an auxiliary regulation error signal. Thus, the control objective can be stated as $\|e(t)\| \rightarrow 0$. Note that the auxiliary error signal $r(t)$ is not measurable, since (15) depends on $\dot{p}(t)$.

\section{A. Open-loop Error System}

In (15), the auxiliary regulation error signal, $e_{f}$, is an output of the dynamic filter [19], [20] as

$$
\begin{aligned}
\dot{q} & =-(k+2 \phi) q-\eta+(k+\phi)^{2} e+e \\
\dot{\eta} & =q-\phi \eta-(k+\phi) e \\
e_{f} & =q-(k+\phi) e
\end{aligned}
$$

where $k \in \mathbb{R}^{2 \times 2}$ is a positive definite, diagonal control gain matrix, $\eta(t) \in \mathbb{R}^{2}$ is another output of the filter, and $q(t) \in \mathbb{R}^{2}$ is an internal filter variable.

After using (15), (16), and (18), the time derivative of $e_{f}(t)$ can be obtained as

$$
\dot{e}_{f}=-\phi e_{f}-\eta+e-(k+\phi) r .
$$

The expression in (19) will be utilized in the subsequent Lyapunov-based stability analysis.

After premultiplying (15) by $M_{s}$, taking the time derivative of the result, and utilizing (6), the open loop error dynamics are obtained as

$$
M_{s} \dot{r}=\tilde{N}(t)+N_{d}(t)+\Omega u_{d}-M_{s}(k+\phi) r-S e+S \phi e_{f}
$$

where $S \in \mathbb{R}^{n \times n}$ is a subsequently defined uncertain matrix (i.e., see Lemma 1 and Assumption 2), and the unknown, unmeasurable auxiliary signals $\tilde{N}(t), N_{d}(t) \in \mathbb{R}^{2}$ are defined as

$$
\begin{gathered}
\tilde{N}(t) \triangleq \chi(t)+M_{s}\left(\phi r+\left(1-\phi^{2}\right) e-2 \phi e_{f}-\eta\right)+S e-S \phi e_{f} \\
N_{d}(t) \triangleq-d(t) .
\end{gathered}
$$

The motivation for separating the terms as in (21) and (22) is based on the fact that the following upper bounds can be developed:

$$
\|\tilde{N}(t)\| \leq \rho(\|z\|)\|z\|, \quad\left\|N_{d}(t)\right\| \leq \zeta_{d 1}, \quad\left\|\dot{N}_{d}(t)\right\| \leq \zeta_{d 2}
$$

where $\rho(\cdot) \in \mathbb{R}$ is a positive, globally invertible non-decreasing function; $\zeta_{d 1}, \zeta_{d 2} \in \mathbb{R}^{+}$are known bounding constants; and $z(t) \in \mathbb{R}^{8}$ is defined as

$$
z(t) \triangleq\left[\begin{array}{llll}
e^{T}(t) & e_{f}^{T}(t) & r^{T}(t) & \eta^{T}(t)
\end{array}\right]^{T} .
$$

A precise definition of the function $\rho(\cdot)$ is not required in the subsequent stability analysis. It is only required to satisfy the aforementioned mathematical properties.

\section{B. Closed-loop Error System}

Based on the open-loop regulation error dynamics in (20) and the subsequent stability analysis, the auxiliary control $u_{d}(t)$ is designed as

$$
u_{d}(t)=\hat{\Omega}^{-1}\left(\mu_{0}-\mu_{1}\right)
$$

where $\hat{\Omega} \in \mathbb{R}^{2 \times 2}$ is a constant, best-guess estimate of the uncertain matrix $\Omega$. In (25), $\mu_{0}(t), \mu_{1}(t) \in \mathbb{R}^{2}$ are subsequently defined feedback control terms.

After substituting (25) into (20), the closed-loop error dynamics are obtained as

$$
M_{s} \dot{r}=\tilde{N}+N_{d}+\tilde{\Omega}\left(\mu_{0}-\mu_{1}\right)-M_{s}(k+\phi) r-S e+S \phi e_{f}
$$

where the constant uncertain matrix $\tilde{\Omega} \in \mathbb{R}^{2 \times 2}$ is defined as

$$
\tilde{\Omega}=\Omega \hat{\Omega}^{-1} .
$$

Lemma 1. Any positive definite matrix $X \in \mathbb{R}^{n \times n}$ can be decomposed as

$$
X=S T,
$$

where $S \in \mathbb{R}^{n \times n}$ is a positive definite symmetric matrix and $T \in \mathbb{R}^{n \times n}$ is an unity upper triangular matrix [21].

Proof. Proof of Lemma 1 can be found in [21] and is omitted here for brevity.

Assumption 2. Bounds on the uncertain matrix $\Omega \in \mathbb{R}^{n \times n}$ are known such that the constant feed forward estimate $\hat{\Omega} \in \mathbb{R}^{n \times n}$ can be chosen to render the product, $\tilde{\Omega}=\Omega \hat{\Omega}^{-1}$ is positive definite. Further, the estimate $\tilde{\Omega}$ is selected such that

$$
\tilde{\Omega}=S T,
$$


where the unity upper triangular matrix $T$ is diagonally dominant in the sense that

$$
\varepsilon \leq\left|T_{i i}\right|-\sum_{k=i+1}^{n}\left|T_{i k}\right| \leq Q, \quad i=1, \ldots, n-1 .
$$

where $\varepsilon \in(0,1)$ and $Q \in \mathbb{R}^{+}$are known bounding constants, and $T_{i k} \in \mathbb{R}$ denotes the $(i, k)^{\text {th }}$ element of the matrix $T$. In (29), the matrices $S$ and $T$ are defined similarly as in Lemma 1.

After utilizing the decomposition in (29), the error dynamics in (26) can be rewritten as

$$
M \dot{r}=\tilde{N}_{1}+N_{d 1}+T\left(\mu_{0}-\mu_{1}\right)-\left(k_{m}+\phi_{m}\right) r-e+\phi e_{f}
$$

where $M \triangleq S^{-1} M_{s}, k_{m} \triangleq S^{-1} M_{s} k$, and $\phi_{m} \triangleq S^{-1} M_{s} \phi$

$$
\tilde{N}_{1} \triangleq S^{-1} \tilde{N}, \quad N_{d 1} \triangleq S^{-1} N_{d} .
$$

Since $S$ is constant and positive definite, $\tilde{N}_{1}(t)$ and $N_{d 1}(t)$ satisfy the inequalities

$$
\left\|\tilde{N}_{1}\right\| \leq \rho_{1}(\|z\|)\|z\|, \quad\left\|N_{d 1}\right\| \leq \zeta_{N_{d 1}}, \quad\left\|\dot{N}_{d 1}\right\| \leq \zeta_{\dot{N}_{d 1}}
$$

where $\rho_{1}(\cdot) \in \mathbb{R}$ is a positive, globally invertible non-decreasing function; $\zeta_{N_{d 1}}, \zeta_{\dot{N}_{d 1}} \in \mathbb{R}^{+}$are known bounding constants. By utilizing the fact that the uncertain matrix $T$ is unity upper triangular, the error dynamics in (31) can be rewritten as

$$
M \dot{r}=\tilde{N}_{1}+N_{d 1}-\left(k_{m}+\phi_{m}\right) r+\mu_{0}+\bar{T} \mu_{0}-T \mu_{1}-e+\phi e_{f}
$$

where $\bar{T} \triangleq T-I_{2 \times 2}$ is a strictly upper triangular matrix, and $I_{2 \times 2}$ denotes a $2 \times 2$ identity matrix. Based on the open-loop error system in (34), the auxiliary control terms $\mu_{0}(t)$ and $\mu_{1}(t)$ are designed as

$$
\begin{aligned}
& \mu_{0}=k e_{f} \\
& \mu_{1}=\beta_{f} \operatorname{sgn}(\phi e+\phi \eta)
\end{aligned}
$$

where $k$ is introduced in (16)-(18); and $\beta_{f} \in \mathbb{R}^{2 \times 2}$ denotes a positive definite, diagonal control gain matrix. After substituting (35) into (34), the closed-loop error system is obtained as

$$
M \dot{r}=\tilde{N}_{1}+\bar{T} \mu_{0}+N_{d 1}-\left(k_{m}+\phi_{m}\right) r-T \mu_{1}+(k+\phi) e_{f}-e .
$$

The term $\bar{T} \mu_{0}$ can be expressed as

$$
\bar{T} \mu_{0}=\left[0, T_{12} ; 0,0\right]\left[\begin{array}{ll}
\mu_{01}(t) & \mu_{02}(t)
\end{array}\right]^{T}=\left[\begin{array}{ll}
T_{12} \mu_{02}(t) & 0
\end{array}\right]^{T}=\left[\begin{array}{ll}
\Lambda_{\rho} & 0
\end{array}\right]^{T}
$$

where $\Lambda_{\rho}$ satisfies the inequality

$$
\left\|\Lambda_{\rho}\right\| \leq \rho_{\Lambda 1}\|z\|
$$

where $z(t)$ is defined in (24), and $\rho_{\Lambda 1} \in \mathbb{R}^{+}$is a known bounding constant.

By utilizing (38), the error dynamics in (37) can be expressed as

$$
M \dot{r}=\tilde{N}_{2}+N_{d 1}-\left(k_{m}+\phi_{m}\right) r-T \mu_{1}+(k+\phi) e_{f}-e
$$

where

$$
\tilde{N}_{2}=\tilde{N}_{1}+\bar{T} \mu_{0}=\tilde{N}_{1}+\left[\begin{array}{ll}
\Lambda_{\rho} & 0
\end{array}\right]^{T} .
$$

Based on (33), (39), and (41), $\tilde{N}_{2}(t)$ satisfies the inequality

$$
\left\|\tilde{N}_{2}\right\| \leq \rho_{2}(\|z\|)\|z\|
$$

where $\rho_{2}(\cdot) \in \mathbb{R}$ is positive, globally invertible nondecreasing function.

To facilitate the following stability analysis, the control gain $\beta_{f}$ introduced in (36) is selected to satisfy the sufficient condition

$$
\beta_{f}>\frac{1}{\varepsilon}\left(\zeta_{N_{d 1}}+\frac{1}{\phi} \zeta_{\dot{N}_{d 1}}\right)
$$

where $\zeta_{N_{d 1}}$ and $\zeta_{\dot{N}_{d 1}}$ are introduced in (33), $\varepsilon$ is introduced in (30), and $\phi$ is introduced in (15).

\section{Stability Analysis}

To facilitate the following stability analysis, let $\mathcal{D} \subset \mathbb{R}^{9}$ be a domain containing $y(t)=0$, where $y(t) \in \mathbb{R}^{9}$ is defined as

$$
y(t) \triangleq\left[\begin{array}{ll}
z^{T}(t) & \sqrt{P(t)}
\end{array}\right]^{T} .
$$


In (44), the non-negative auxiliary function $P(t) \in \mathbb{R}$ is the generalized solution to the differential equation

$$
\begin{gathered}
\dot{P}(t)=-L(t) \\
P(0)=\beta_{f} Q|e(0)+\eta(0)|-(e(0)+\eta(0))^{T} N_{d 1}(0)
\end{gathered}
$$

where the auxiliary function $L(t) \in \mathbb{R}$ is defined as

$$
L(t) \triangleq r^{T}(t)\left(N_{d 1}(t)-T \mu_{1}(t)\right) .
$$

Lemma 2. Provided the sufficient condition in (43) is satisfied, the following inequality can be obtained:

$$
\int_{0}^{t} L(\tau) d \tau \leq \beta_{f} Q|e(0)+\eta(0)|-(e(0)+\eta(0))^{T} N_{d 1}(0) .
$$

Hence, (45), (46), and (48) can be used to conclude that $P(t) \geq 0$.

Proof of Lemma 2 can be found in the appendix.

Theorem 1. The robust output feedback control law given in (14), (16), (17), (18), and (25) ensures asymptotic regulation of LCO in the sense that

$$
\|e(t)\| \rightarrow 0 \quad \text { as } \quad t \rightarrow \infty
$$

provided the control gain, $k$, introduced in (35) is selected sufficiently large (see the subsequent proof), and $\beta_{f}$ is selected to satisfy the sufficient condition in (43).

Proof. Let $V(y, t): \mathcal{D} \times[0, \infty) \rightarrow \mathbb{R}$ be a non-negative function defined as

$$
V=\frac{1}{2} e^{T} e+\frac{1}{2} e_{f}^{T} e_{f}+\frac{1}{2} r^{T} M r+\frac{1}{2} \eta^{T} \eta+P
$$

which satisfies the inequalities

$$
U_{1}(y) \leq V(y, t) \leq U_{2}(y)
$$

provided the sufficient condition in (43) is satisfied. In (51), the continuous positive definite functions $U_{1}(y), U_{2}(y) \in \mathbb{R}$ are defined as

$$
U_{1}(y) \triangleq \eta_{1}\|y\|^{2}, \quad U_{2}(y) \triangleq \eta_{2}\|y\|^{2}
$$

where $\eta_{1}, \eta_{2} \in \mathbb{R}$ are defined as

$$
\eta_{1} \triangleq \frac{1}{2} \min \left\{1, \lambda_{\min }(M)\right\}, \quad \eta_{2} \triangleq \max \left\{\frac{1}{2} \lambda_{\max }(M), 1\right\}
$$

where $\lambda_{\min }(\cdot), \lambda_{\max }(\cdot)$ denote the minimum and maximum eigenvalues of the arguments, respectively. After taking the time derivative of (50) and utilizing (15), (16), (17), (18), (19), (26), (45), and (47), $\dot{V}(t)$ can be expressed as

$$
\begin{aligned}
\dot{V} & =e^{T}\left(r-\phi e-e_{f}\right)+e_{f}^{T}\left(e-\phi e_{f}-\eta-(k+\phi) r\right) \\
& +r^{T}\left(\tilde{N}_{2}+N_{d 1}-T \mu_{1}-\left(k_{m}+\phi_{m}\right) r+(k+\phi) e_{f}-e\right)+\eta^{T}\left(e_{f}-\phi \eta\right)-r^{T}\left(N_{d 1}-T \mu_{1}\right)
\end{aligned}
$$

where the symmetry of $M=S^{-1} M_{s}$ was utilized. After canceling common terms in (53), $\dot{V}(t)$ can be simplified as

$$
\dot{V}=-\phi\|e\|^{2}-\phi\left\|e_{f}\right\|^{2}-\phi\|\eta\|^{2}-\phi_{m}\|r\|^{2}+r^{T}\left(\tilde{N}_{2}-k_{m} r\right) \text {. }
$$

After using the bounding inequality in (42), the expression in (54) can be upper bounded as

$$
\dot{V} \leq-\lambda_{0}\|z\|^{2}-\left(k_{m}\|r\|^{2}-\rho_{2}(\|z\|)\|r\|\|z\|\right)
$$

where $\lambda_{0} \triangleq \min \left\{\lambda_{\min }(\phi), \lambda_{\min }\left(\phi_{m}\right)\right\}$. By completing the squares for the last two terms in (55), the upper bound on $\dot{V}(t)$ can be expressed as

$$
\dot{V} \leq-\left(\lambda_{0}-\frac{\rho_{2}^{2}(\|z\|)}{4 k_{m}}\right)\|z\|^{2} .
$$

The following expression can be obtained from (56):

$$
\dot{V} \leq-U(y)
$$

where $U(y)=c\|z\|^{2}$, for some positive constant $c \in \mathbb{R}$ is a continuous positive semi-definite function that is defined on the domain

$$
\mathcal{D} \triangleq\left\{y(t) \in \mathbb{R}^{9} \mid\|y\| \leq \rho^{-1}\left(2 \sqrt{k_{m} \lambda_{0}}\right)\right\} .
$$


The expressions in (51) and (57) can be used to prove that $e(t), e_{f}(t), r(t), \eta(t) \in \mathcal{L}_{\infty}$ in $\mathcal{D}$. Given that $e(t), e_{f}(t)$, $r(t), \eta(t) \in \mathcal{L}_{\infty},(15)$ can be used to show that $\dot{e}(t) \in \mathcal{L}_{\infty}$ in $\mathcal{D}$; (19) can be used to show that $\dot{e}_{f}(t) \in \mathcal{L}_{\infty}$ in $\mathcal{D}$; and (16) can be used to show that $q(t) \in \mathcal{L}_{\infty}$ in $\mathcal{D}$. It then follows that $\dot{\eta}(t) \in \mathcal{L}_{\infty}$ in $\mathcal{D}$ from (17). Given that $e_{f}(t) \in \mathcal{L}_{\infty}$ the expressions in (25), (35), and (36) can be used along with (10) and (11) to prove that the control signals $u_{d i}(t), v_{i}(t)$, $u_{i}(t) \in \mathcal{L}_{\infty}$ in $\mathcal{D}$. Since $e(t), e_{f}(t), r(t), \eta(t) \in \mathcal{L}_{\infty},(33),(40)$, and (42) can be used to prove that $\dot{r}(t) \in \mathcal{L}_{\infty}$ in $\mathcal{D}$. Given that $\dot{e}(t), \dot{e}_{f}(t), \dot{r}(t), \dot{\eta}(t) \in \mathcal{L}_{\infty}$, it follows that $e(t), e_{f}(t), r(t)$, and $\eta(t)$ are uniformly continuous in $\mathcal{D}$; thus, $z(t)$ is uniformly continuous throughout closed-loop controller operation. Hence, the definitions of $U(y)$ and $z(t)$ can be used to prove that $U(y)$ is uniformly continuous in $\mathcal{D}$.

Let $\mathcal{S} \subset \mathcal{D}$ denote a set defined as follows:

$$
\mathcal{S} \triangleq\left\{y(t) \subset \mathcal{D} \mid U(y(t)) \leq \eta_{1}\left(\rho^{-1}\left(2 \sqrt{k_{m} \lambda_{0}}\right)\right)^{2}\right\}
$$

Theorem 8.4 of [22] can now be invoked to state that

$$
c\|z(t)\|^{2} \rightarrow 0 \quad \text { as } \quad t \rightarrow \infty \quad \forall y\left(t_{0}\right) \in \mathcal{S} .
$$

Based on the definition of $z(t)$, (59) can be used to show that

$$
\|e(t)\| \rightarrow 0 \quad \text { as } \quad t \rightarrow \infty \quad \forall y\left(t_{0}\right) \in \mathcal{S} .
$$

Thus, asymptotic regulation of the pitching and plunging displacements can be achieved, provided the initial conditions are within the set $\mathcal{S}$, where $\mathcal{S}$ can be made arbitrarily large by increasing the control gain $k$ (recall that $k_{m}=S^{-1} M_{s} k$ ). Hence, this is a semi-global asymptotic result.

\section{Simulation Results}

A numerical simulation was created to demonstrate the performance of the proposed control law. In order to develop a realistic stepping stone to high-fidelity numerical simulation results using detailed CFD models, the following simulation results are based on detailed dynamic parameters and specifications. The simulation is based on the dynamic model given in (1)-(5). The dynamic parameters utilized in the simulation are summarized in Table I and were obtained from [16]. The actual parameters $\theta_{1}^{*}$ and $\theta_{2}^{*}$ and their estimates $\hat{\theta}_{1, i}$ and $\hat{\theta}_{2, i}$ are described in Table II were obtained from [12]. The following simulation results were

TABLE I

DYNAMIC PARAMETERS AND GEOMETRIC DIMENSIONS OF THE WING SECTION

TABLE II

SJA PARAMETERS AND THEIR ESTIMATES

\begin{tabular}{|l|l|l|}
\hline$\rho=1.225 \mathrm{~kg} / \mathrm{m}^{3}$ & $a=-0.6$ & $c_{m_{\alpha}}=-0.635$ \\
\hline$m=12.387 \mathrm{~kg}$ & $b=0.125 \mathrm{~m}$ & $v=13 \mathrm{~m} / \mathrm{s}$ \\
\hline$C_{\alpha}=0.036 \mathrm{~kg} \cdot \mathrm{m}^{2} / s$ & $c_{l_{\beta}}=3.358$ & $s_{p}=0.6 \mathrm{~m}$ \\
\hline$I_{\alpha}=0.065 \mathrm{~kg} \cdot \mathrm{m}$ & $C_{h}=27.43 \mathrm{~kg} / \mathrm{s}$ & $c_{l_{\alpha}}=6.28$ \\
\hline$K_{h}=2844.4 \mathrm{~N} / \mathrm{m}$ & $c_{m_{\beta}}=-0.635$ & $x_{a}=0.2847$ \\
\hline
\end{tabular}

\begin{tabular}{|l|l|l|l|}
\hline$\theta_{1, i}^{*}$ & $\hat{\theta}_{1, i}$ & $\theta_{2, i}^{*}$ & $\hat{\theta}_{2, i}$ \\
\hline 32.9 Volt-deg & 32.7 Volt-deg & $16.5 \mathrm{deg}$ & $16.4 \mathrm{deg}$ \\
\hline 29.8 Volt-deg & 29.7 Volt-deg & $15.9 \mathrm{deg}$ & $15.8 \mathrm{deg}$ \\
\hline
\end{tabular}

obtained using control gains selected as

$$
\beta_{f}=\operatorname{diag}(0.68,0.178), \quad k=\operatorname{diag}(1,3.55), \quad \phi=\operatorname{diag}(0.05,5) .
$$

The control gains given in (60) were selected based on achieving a desirable response in terms of settling time and required control effort. To test the case where the input gain matrix $B$ is uncertain, it is assumed in the simulation that the actual value of $B$ is the $2 \times 2$ identity matrix, but the constant feedforward estimate $\hat{B}$ used in the control law is given by

$$
\hat{B}=\left[\begin{array}{cc}
0.9 & 0.1 \\
-0.1 & 1.1
\end{array}\right]
$$

The initial conditions used in the simulation are

$$
p(0)=\left[\begin{array}{ll}
0.02 & 0.2
\end{array}\right]^{T}
$$

The external disturbance used in the simulation is

$$
d=0.01[0.003 \sin (0.5 t) \quad 0.003 \sin (0.5 t)+0.001 \sin (0.5 t)]^{T}
$$

Fig. 1 shows that the open-loop system exhibits pitching and plunging LCO. Figs. 2 - 4 show the performance of the proposed control law to regulate the pitching and plunging LCO. The figures show the performance of the control law for 10 different sets of off-nominal values of the uncertain SJA parameters $\theta_{1}^{*}$ and $\theta_{2}^{*}$ using a Monte Carlo-type simulation. In each of the 10 trials, the values of the uncertain parameters were generated randomly to test a range of uncertainty of $\pm 1 \%$ off nominal. Fig. 3 shows the virtual surface deflection angle control inputs during closed-loop controller operation, and Fig. 4 shows the corresponding SJA control voltage inputs in the closed-loop for the 10 trials. The commanded control inputs remain within reasonable limits throughout the duration of the simulation for all 10 trials. 

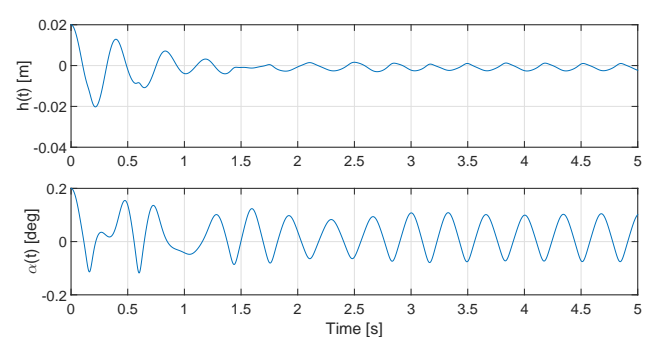

Fig. 1. Open loop plunging and pitching displacements of the LCO response in the simulated system.
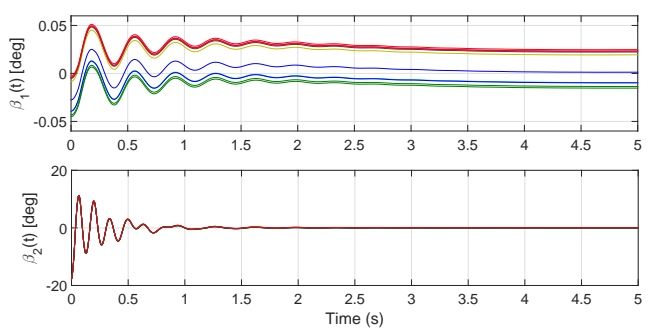

Fig. 3. Virtual deflection angle $\beta(t)$ control commands during closed-loop operation for 10 sets of values of the SJA parameters $\theta_{1}^{*}$ and $\theta_{2}^{*}$.
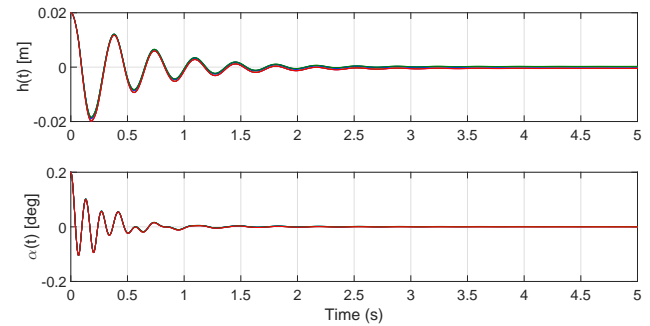

Fig. 2. Closed-loop regulation of pitching and plunging LCO using the proposed control law for 10 different off-nominal sets of values for $\theta_{1}^{*}$ and $\theta_{2}^{*}$.
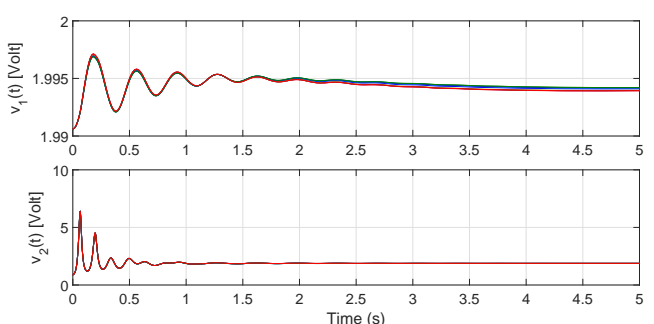

Fig. 4. SJA control voltage input signals commanded during closed-loop operation for the 10 different trials.

\section{Conclusions}

A SJA-based output feedback control method is presented, which achieves asymptotic LCO regulation in small unmanned aerial vehicle (SUAV) wings in the presence of uncertain SUAV dynamics and unmodeled external disturbances. In addition, the proposed control method compensates for the parametric uncertainty and nonlinearity inherent in the SJA actuator dynamics. Motivated by the limitations characteristic of SUAV applications, the control method is designed to be computationally inexpensive, eliminating the need for time-varying parameter update laws, function approximators, or heavy computations. To achieve the result, a robust-inverse control method is utilized, which is proven to compensate for the SUAV and SJA uncertainties using a simplified controller structure. By endowing the robust-inverse control structure with with a bank of dynamic filters, asymptotic LCO regulation is achieved using only pitching and plunging displacement measurements in the feedback loop. The result is an asymptotic SJA-based LCO regulation control design, which does not require velocity measurements, adaptive laws, function approximators, or heavy computations in the feedback loop. A rigorous Lyapunov-based stability analysis is utilized to prove the theoretical result, and numerical simulation results demonstrate that the proposed control law asymptotically regulates LCO in the presence of significant SJA parameter uncertainty.

\section{ACKNOWLEDGMENT}

This research is supported in part by NSF award number 1335405.

\section{APPENDIX}

Proof of Lemma 2 Provided the sufficient gain condition in (43) is satisfied, the following inequality can be obtained:

$$
\int_{0}^{t} L(\tau) d \tau \leq \beta_{f} Q|e(0)+\eta(0)|-(e(0)+\eta(0))^{T} N_{d 1}(0) \in \mathbb{R}
$$

Hence, (62) can be used to conclude that $P(t) \geq 0$, where $P(t)$ is defined in (45) and (46).

To facilitate the following proof, the expression in (47) will be rewritten in a more advantageous form as follows:

$$
L(t)=\sum_{i=1}^{m}\left(r_{i}(t)\left(N_{d 1 i}(t)-\sum_{j=i}^{m} T_{i j} \mu_{1 j}(t)\right)\right) \in \mathbb{R}
$$

where $m=2$ for the LCO regulation objective in the current result. In (63), $r_{i}(t), N_{d 1 i}(t), \mu_{1 i}(t) \in \mathbb{R}$ for $i=1, \ldots, m$ denote the $i^{t h}$ elements of the vectors $r(t), N_{d 1}(t)$, and $\mu_{1}(t)$; and $T_{i j} \in \mathbb{R}$ for $i=1, \ldots, m$ and $j=1, \ldots m$ denote the $(i, j)^{t h}$ elements of the matrix $T$. 
Proof. Integrating both sides of (63) yields

$$
\int_{0}^{t} L(\tau) d \tau=\int_{0}^{t} \sum_{i=1}^{m} r_{i}(\tau)\left(N_{d 1 i}(\tau)-\sum_{j=i}^{m} T_{i j} \mu_{1 j}(\tau)\right) d \tau \in \mathbb{R} .
$$

Based on the expressions in (15)-(19), the integral in (64) can be expressed as

$$
\begin{aligned}
\int_{0}^{t} L(\tau) d \tau= & \int_{0}^{t} \sum_{i=1}^{m}\left(\dot{e}_{i}(\tau)+\dot{\eta}_{i}(\tau)\right)\left(N_{d 1 i}(\tau)-\sum_{j=i}^{m} T_{i j} \mu_{1 j}(\tau)\right) d \tau \\
& +\int_{0}^{t} \sum_{i=1}^{m}\left(\phi_{i} e_{i}(\tau)+\phi_{i} \eta_{i}(\tau)\right)\left(N_{d 1 i}(\tau)-\sum_{j=i}^{m} T_{i j} \mu_{1 j}(\tau)\right) d \tau
\end{aligned}
$$

where $\phi_{i} \in \mathbb{R}$ denotes the $i^{\text {th }}$ diagonal element of the control gain matrix $\phi$. By defining an auxiliary variable $\nu(t) \in \mathbb{R}^{m}$ as

$$
\nu(t) \triangleq e(t)+\eta(t)
$$

the expression in (65) can be rewritten as

$$
\begin{aligned}
\int_{0}^{t} L(\tau) d \tau= & \int_{0}^{t} \sum_{i=1}^{m} \frac{\partial \nu_{i}(\tau)}{\partial \tau} N_{d 1 i}(\tau) d \tau-\int_{0}^{t} \sum_{i=1}^{m} \frac{\partial \nu_{i}(\tau)}{\partial \tau} \sum_{j=i}^{m} T_{i j} \mu_{1 j}(\tau) d \tau \\
& +\int_{0}^{t} \sum_{i=1}^{m} \phi_{i} \nu_{i}(\tau)\left(N_{d 1 i}(\tau)-\sum_{j=i}^{m} T_{i j} \mu_{1 j}(\tau)\right) d \tau
\end{aligned}
$$

By evaluating the first integral in (67) using integration by parts, Equation (67) can be expressed as

$$
\begin{aligned}
\int_{0}^{t} L(\tau) d \tau= & \sum_{i=1}^{m} \nu_{i}(t) N_{d 1 i}(t)-\sum_{i=1}^{m} \nu_{i}(0) N_{d 1 i}(0)-\int_{0}^{t} \sum_{i=1}^{m} \nu_{i}(\tau) \frac{\partial N_{d 1 i}(\tau)}{\partial \tau} d \tau \\
& -\int_{0}^{t} \sum_{i=1}^{m} \frac{\partial \nu_{i}(\tau)}{\partial \tau} \sum_{j=i}^{m} T_{i j} \mu_{1 j}(\tau) d \tau+\int_{0}^{t} \sum_{i=1}^{m} \phi_{i} \nu_{i}(\tau)\left(N_{d 1 i}(\tau)-\sum_{j=i}^{m} T_{i j} \mu_{1 j}(\tau)\right) d \tau .
\end{aligned}
$$

After substituting the definition of the auxiliary control term $\mu_{1}(t)$ given in (36) and rearranging, (68) can be expressed as

$$
\begin{aligned}
\int_{0}^{t} L(\tau) d \tau= & \sum_{i=1}^{m} \nu_{i}(t) N_{d 1 i}(t)-\sum_{i=1}^{m} \nu_{i}(0) N_{d 1 i}(0)-\int_{0}^{t} \sum_{i=1}^{m} \frac{\partial \nu_{i}(\tau)}{\partial \tau} \sum_{j=i}^{m} T_{i j} \beta_{f} \operatorname{sgn}\left(\phi_{j} \nu_{j}(\tau)\right) d \tau \\
& +\int_{0}^{t} \sum_{i=1}^{m} \phi_{i} \nu_{i}(\tau)\left(N_{d 1 i}(\tau)-\frac{1}{\phi} \frac{\partial N_{d 1 i}(\tau)}{\partial \tau}-\sum_{j=i}^{m} T_{i j} \beta_{f} \operatorname{sgn}\left(\phi_{j} \nu_{j}(\tau)\right)\right) d \tau .
\end{aligned}
$$

By using the fact that

$$
\sum_{j=i}^{m} T_{i j} \beta_{f} \operatorname{sgn}\left(\phi_{j} \nu_{j}(\tau)\right)=\beta_{f}\left(\operatorname{sgn}\left(\phi_{j} \nu_{j}(\tau)\right)+\sum_{j=i+1}^{m} \bar{T}_{i j} \operatorname{sgn}\left(\nu_{j}(\tau)\right)\right)
$$

the bounding inequalities in (30) can be used to express (69) as

$$
\begin{aligned}
\int_{0}^{t} L(\tau) d \tau= & \sum_{i=1}^{m} \nu_{i}(t) N_{d 1 i}(t)-\sum_{i=1}^{m} \nu_{i}(0) N_{d 1 i}(0)-\int_{0}^{t} \sum_{i=1}^{m} \frac{\partial \nu_{i}(\tau)}{\partial \tau} \beta_{f} \delta \operatorname{sgn}\left(\phi_{i} \nu_{i}(\tau)\right) d \tau \\
& +\int_{0}^{t} \sum_{i=1}^{m} \phi_{i} \nu_{i}(\tau)\left(N_{d 1 i}(\tau)-\frac{1}{\phi} \frac{\partial N_{d 1 i}(\tau)}{\partial \tau}-\delta \beta_{f} \operatorname{sgn}\left(\phi_{i} \nu_{i}(\tau)\right)\right) d \tau
\end{aligned}
$$

where $\delta \in(\varepsilon, Q)$ is a positive constant parameter. By using the property

$$
\int_{0}^{t} \frac{\partial \nu(\tau)}{\partial \tau} \operatorname{sgn}(\phi \nu(\tau)) d \tau=|v(t)|-|v(0)|
$$

the expression in (71) can be rewritten as

$$
\begin{aligned}
\int_{0}^{t} L(\tau) d \tau= & -\sum_{i=1}^{m} \nu_{i}(0) N_{d 1 i}(0)+\sum_{i=1}^{m} \beta_{f} \delta\left|v_{i}(0)\right|+\sum_{i=1}^{m} \nu_{i}(t) N_{d 1 i}(t)-\sum_{i=1}^{m} \beta_{f} \delta\left|v_{i}(t)\right| \\
& +\int_{0}^{t} \sum_{i=1}^{m} \phi_{i} \nu_{i}(\tau)\left(N_{d 1 i}(\tau)-\frac{1}{\phi} \frac{\partial N_{d 1 i}(\tau)}{\partial \tau}-\delta \beta_{f} \operatorname{sgn}\left(\phi_{i} \nu_{i}(\tau)\right)\right) d \tau
\end{aligned}
$$


The expression in (73) can be upper bounded as

$$
\begin{aligned}
\int_{0}^{t} L(\tau) d \tau \leq & -\nu^{T}(0) N_{d 1}(0)+\beta_{f} Q|\nu(0)|+\sum_{i=1}^{m}\left(\zeta_{N_{d 1}}-\varepsilon \beta_{f}\right)\left|\nu_{i}(t)\right| \\
& +\int_{0}^{t} \sum_{i=1}^{m} \phi_{i}\left|\nu_{i}(\tau)\right|\left(\zeta_{N_{d 1}}-\frac{1}{\phi} \zeta_{\dot{N}_{d 1}}-\varepsilon \beta_{f}\right) d \tau .
\end{aligned}
$$

Thus, it is clear from (74) that if $\beta_{f}$ satisfies the sufficient condition in (43), then

$$
\int_{0}^{t} L(\tau) d \tau \leq \beta_{f} Q|e(0)+\eta(0)|-(e(0)+\eta(0))^{T} N_{d 1}(0) .
$$

Hence, $P(t) \geq 0$ from (45), (46), and (75).

\section{REFERENCES}

[1] P. Yang, Q. D. Li, Z. Ren, Q. K. Tan, and Y. Fan, "Active control method on flutter suppression of a high-aspect-ratio two-dimensional airfoil with a control surface," in Proceedings of 2014 IEEE Chinese Guidance, Navigation and Control Conference, Yantai, China, August 2014, pp. 2745-2752.

[2] G. Platanitis and T. W. Strganac, "Control of a nonlinear wing section using leading- and trailing-edge surfaces," Journal of Guidance, Control, and Dynamics, vol. 27, no. 1, pp. 52-58, 2004.

[3] M. Zardel and F. Bakhtiari-Nejad, "Limit cycle oscillation control of wing with static output feedback control method," Aerospace Science and Technology, vol. 24, pp. 147-160, 2013.

[4] X.-J. Liu, F. Lara-Rosano, and C. W. Chan, "Model-reference adaptive control based on neurofuzzy networks," IEEE Transactions on Systems, Man, and Cybernetics C, vol. 34, no. 3, pp. 302-309, Aug. 2004.

[5] Z. Sun, S. Haghighat, H. H.T. Liu, and J. Bai, "Time-domain modeling and control of a wing-section stall flutter," Journal of Sound and Vibration, vol. 340, pp. 221-238, 2015.

[6] D. Li, J. Xiang, and S. Guo, "Adaptive control of a nonlinear aeroelastic system," Aerospace Science and Technology, vol. 25, pp. 343-352, 2011.

[7] K. V. Singh, "Active aeroelastic control with time delay for targeted flutter modes," Aerospace Science and Technology, vol. 43, pp. 281-288, 2015.

[8] D. W. Wu, M. Chen, H. J. Gong, and H. Ye, "Control of wing rock based on high order sliding mode disturbance observer," in Proceedings of the 11th World Congress on Intelligent Control and Automation, Shenyang, China, June 2014, pp. 873-878.

[9] Z. J. J. Li, M. Wang and G. Dimirovski, "Output feedback control for discrete-time systems with faulty actuators based on average dwell-time method," in Proceedings of the 33rd Chinese Control Conference, Nanjing, China, Dec 2014, pp. 4125-4130.

[10] K. P. A. K. Bondhus and J. T. Gravdahl, "Leader/follower synchronization of satellite attitude without angular velocity measurements," in Proceedings of the 44th IEEE Conference on Decision and Control, and the European Control Conference 2005, Seville, Spain, July 2005, pp. 7270-7277.

[11] M. Amitay, M. Horvath, M. Michaux, and A. Glezer, "Virtual aerodynamic shape modification at low angles of attack using synthetic jet actuators," AIAA Paper 2001-2975, 2001.

[12] W. MacKunis, S. Subramanian, S. Mehta, J. W. Curtis, M. Reyhanoglu, and C. Ton, "Robust nonlinear aircraft tracking control using synthetic jet actuators," in IEEE Conference on Decision and Control (CDC), Dec. 2013, pp. 220-225.

[13] D. Deb, G. Tao, J. O. Burkholder, and D. R. Smith, "Adaptive synthetic jet actuator compensation for a nonlinear tailless aircraft model at low angles of attack," in American Control Conference, June 2006.

[14] - , "Adaptive compensation control of synthetic jet actuator arrays for airfoil virtual shaping," Journal of Aircraft, vol. 44, no. 2, pp. 616-626, Mar.-Apr. 2007.

[15] — - "Adaptive synthetic jet actuator compensation for a nonlinear aircraft model at low angles of attack," IEEE Transactions on Control Systems Technology, vol. 16, no. 5, pp. 983-995, Sept. 2008.

[16] M. R. Elhami and M. F. Narab, "Comparison of sdre and smc control approaches for flutter suppression in a nonlinear wing section," in American Control Conference, June 2012, pp. 6148-6153.

[17] S. N. Singh and W. Yim, "State feedback control of an aero-elastic system with nonlinearity," Journal of the Aerospace Sciences, vol. 7, pp. 23-31, 2003.

[18] S. T. Mondschein, G. Tao, and J. O. Burkholder, "Adaptive actuator nonlinearity compensation and disturbance rejection with an aircraft application,” in American Control Conference, June-July 2011, pp. 2951-2956.

[19] B. Xian, D. M. Dawson, M. S. de Queiroz, and J. Chen, "A continuous asymptotic tracking control strategy for uncertain nonlinear systems," IEEE Transactions on Automatic Control, vol. 49, no. 7, pp. 1206-1211, July 2004.

[20] H. Dinh, S. Bhasin, D. Kim, and W. E. Dixon, "Dynamic neural network-based global output feedback tracking control for uncertain second-order nonlinear systems," in Proceedings of 2012 American Control Conference, Montreal, Quebec, June 2012, pp. 6418-6423.

[21] A. S. Morse, "A gain matrix decomposition and some of its properties," Systems and Control Letters, vol. 21, no. 1, pp. 1-10, July 1993.

[22] H. K. Khalil, Nonlinear Systems, 3rd ed. Upper Saddle River, NJ: Prentice Hall, 2002. 